\title{
Two Suns? Data Doppelgangers and the Construction of the Digital Self
}

One advantage of our day is that you never have to be more than slightly clever or slightly nice.

Anything more will arouse suspicion and rage, and confound the software that runs the country.

- Lights Out in Wonderland

...Big Tech overlords now have the capacity of modifying perceptions and behavior of people both for profit and control. The massive amount of personal data is the most valuable natural resource of the new economy. People are not the customers anymore, they are the product, and in this metaphysical shift we will not be very different from the bonded servants of the feudal world.

- Ramon Bleuca

In this chapter, there is a consideration of space or spaces and the self. So much of what has been written applies to outer space or the space 
immediately above the earth where satellites and drones fly. But the algorithmic state exists in several forms of space- personal space and terrestrial space as well as cyberspace and outer space.

Earlier the idea that we might be looking at techno feudalism was advanced and Bleuca makes a comparable point above. Are the spaces outlined here the bondage context for the new feudalism, the new political economy which is a version of the political economy found in feudalism? Or are we looking at a new animal, something we are not sure how to describe?

We might try to work this out with respect to the person and the nation state. When we talk about the person in these four kinds of space are we talking about the disregard, except where occasionally necessary, of the boundaries of the nation-state? The latter usually involve terrestrial boundaries, an area of sea off the coast of the land space and the skies above that land space up to a certain height. The spaces discussed are, in a sense, spaces of data. And as we find ourselves in these spaces are we also talking about the disregard of traditional boundaries of the self as we are defined, sometimes in a less than comprehensive or, as theorists discussed below imply, a less than 'thick' way? Where is the self here? In the world of data, how do we define the dimensions of the self, of the collapsing and rephrasing of individuality? 
To begin with we might ask what are people entertained by and what are the role models of the self that they see and hear? The power of Big Tech as with Amazon's ownership of Prime and the way entertainment can be subtly and not so subtly modified by the owners, so to speak, of the theatre matters as pointed out earlier in the discussion of feudalism. In Five the way belief systems are maintained in church or in the city square or in rituals in and out of church in feudal society was compared to the entertainment traps afforded people by the tech giants in what is now called techno feudalism.

The discussion below moves across considerations of the individual and the algorithm, across personal, cyber and outer space to focus on the self. We might start with Alexa as a motherboard and go on to Amazon Sidewalk connecting us wherever we are in a mesh network. Amazon Sidewalk connects Echo and Ring Security devices in such a way as to extend networks beyond the home to the street and the neighbourhood. The Ring operates as a bridge. It would seem that it only works properly if everybody in the neighbourhood joins the mesh. To opt out of Amazon Sidewalk you might need to go to Alexa and toggle it off as it is on automatically.

There is a tension between privacy and convenience in Amazon Sidewalk. The system is encrypted so you don't learn when your neighbour's mail arrives. This aside, if such a webbing of a community can be put 
aside, we are, once again, giving Amazon data this time of a very personal nature as in data about our living space.

At the same time we might ask, why is Amazon doing this? How will such data as Amazon gets from such a mesh help it? Is it to help Amazon delivery trucks logistically? In a sense all and any data is useful for triangulation, cross reference and accuracy even if only occasionally used. As suggested in earlier comments about Bezos in the ongoing review of the book Bezonomics by Brian Dumaine throughout the Two Suns? series it seems to be a matter of finding a gap and then filling it rather than following an overall Master Plan. On the other hand the neighbourhood does seem to be a place to occupy if only because that is where people actually live, it is their living space as distinct from their position in cyberspace.

And then it might be asked, is the ownership of a neighbourhood mesh a trust builder for residents? Is Amazon Sidewalk, as something accepted, 'as part of the furniture', something for Amazon to standardize around? Once a neighbourhood mesh is established it might not be of much use on its own to Amazon but it gains a space that other entities are not so far in that may be useful in future and it feeds Alexa by giving an information edge, as it were, to the immediate locality. Alexa can listen in the house to begin with and now it can listen at the door and on the porch via the ring and to the sounds of 
the street at the letterbox. Then it links to the information without directly hearing it from every other Alexa in every house and every street in the mesh.

Why are state, city or national governments not providing such a mesh? Does the individual not have a right to information about his community? Is this a case of the algorithmic state slipping through the cracks of the nation-state? Is this all because of a gap in the law so that where citizens' rights ought to be protected in their neighbourhoods they are not? Is this like the situation described by Lina Khan regarding anti-trust law where Big Tech just seems to slip through? Are state, city and national governments simply not attuned to the internet of things in an alignment with the rights and information needs of citizens?

Is this a soft invasion by Amazon? Sold as promoting 'a smart neighbourhood'. Sold by the cuteness of keeping track of the dog? Is Amazon grabbing the public space with the velvet gloves of soft power? Is this a takeover of community sovereignty by Big Tech?

The point might be that Amazon is in the space of the family with the door and the mailbox. This is physical, personal, family space and not a matter of just cyberspace. Amazon is grabbing the locale, securing the neighbourhood, proceeding from the nearness of Alexa to the close proximity of the gate to the closer point of the door as the mesh widens to 
the sides and neighbours join in an apparently discreet way so that they do nor share information amongst themselves even as they allow Amazon access to their data.

Some systems go further into the privacy of the individual. Google Hello doorbells have Face Recognition which is not legal in certain states in the USA. This was discussed in Six in terms of the history of facial recognition. There is considerable difference across countries. In China, for example, Facial Recognition is practised widely and legally.

Coming back to ideas of space and the four kinds of space mentioned at the outset of this chapter, personal space, terrestrial space, cyberspace and outer space, there is a contrast of close and far here between the shells of near outer space like Amazon's zone for its Kuiper satellites, its shell in space, and the neighbourhood mesh, the shell, if you like, of the locale.

As well as national governance, governance by the nation state, we might talk about community governance in the case of the neighbourhood mesh. Then there is the governance of the screen, the individual being shaped and influenced by such forms of governance. Regarding screen governance we might ask about Trump and TikTok. How did that go? Does it show a counter example where nation state governance beats Big Tech governance? Or does it show the difficulty involved in such a governance? 
Earlier, in other publications, I have suggested the idea of a remote self where social interaction is mediated through screens and at a distance between individuals (cf Books and Books 31). Here the self exists in the context of a neighbourhood mesh on a day to day basis with Alexa to hand as a ready sounding board for experience and information. A person comes inside the house after walking the dog leaving the neighbourhood mesh only to sit in front of a computer or phone screen and order a pizza by drone delivery through a communications system that extends to the Kuiper satellite system in the Amazon shell. The drone enters the neighbourhood mesh at the top of a power pole in the near vicinity, the pizza is lowered into an autonomous vehicle which proceeds, as it were, through the neighbourhood mesh to be delivered at the door.

Regarding the informed or, for that matter, misinformed self and the general feed of information to the individual from the net, to what extent is that information and experience true and correct and to what extent might it be rigged? How and why is it trusted? We might turn to a quartet of commentators, Tricia Wang, Cathy O'Neill, Molly Sauter and Janet Vertesi. This group offer a importanta set of coping mechanisms for the self in the context of Big Tech. Rather like the history 
outlined in Six their voices are not heard to the extent that they might be.

To begin with the idea as put forward by Tricia Wang, a technology ethnographer, that underneath everything perhaps we are looking at an oracle:

...we have a new oracle, and it's name is big data, or we call it "Watson" or "deep learning" or "neural net." And these are the kinds of questions we ask of our oracle now, like, "What's the most efficient way to ship these phones from China to Sweden?" Or, "What are the odds of my child being born with a genetic disorder?" Or, "What are the sales volume we can predict for this product?"

We use the oracle for the most basic of things:

I have a dog. Her name is Elle, and she hates the rain. And I have tried everything to untrain her. But because I have failed at this, I also have to consult an oracle, called Dark Sky, every time before we go on a walk, for very accurate weather predictions in the next 10 minutes. She's so sweet. So because of all of this, our oracle is a $\$ 122$ billion industry.

Now, despite the size of this industry, the returns are surprisingly low.

Wang discusses the limitations of the oracle and asks: 
...why is having more data not helping us make better decisions, especially for companies who have all these resources to invest in these big data systems? Why isn't it getting any easier for them?

As mentioned in Six, Tricia Wang falls back on personal experience when she found than amongst the working people she knew:

...I found out that the ads the actually enticed them the most were the ones for iPhones, promising them this entry into this high-tech life... I saw people investing over half of their monthly income into buying a phone, and increasingly, they were "shanzhai," which are affordable knock-offs of iPhones and other brands.

The idea of participation in a smart world is incredibly strong and important in a notion of self. Tricia Wang also points out, in ways that correspond with the arguments of O'Neill and Sauter about modelling for humans, that the use of big data may be flawed:

...I started to realize that even the poorest in China would want a smartphone, and that they would do almost anything to get their hands on one.

Wang states that her employer Nokia could not get this basic data point that people wanted to but smart phones for reasons of status and identity: 
...Nokia was not convinced, because it wasn't big data.

But, according to Tricia Wang it's all about the way some systems are contained and others, especially those of a human kind, are not:

Big data's reputation for success comes from quantifying very specific environments, like electricity power grids or delivery logistics or genetic code, when we're quantifying in systems that are more or less contained.

But not all systems are as neatly contained. When you're quantifying and systems are more dynamic, especially systems that involve human beings, forces are complex and unpredictable, and these are things that we don't know how to model so well. Once you predict something about human behavior, new factors emerge, because conditions are constantly changing. That's why it's a neverending cycle. You think you know something, and then something unknown enters the picture. And that's why just relying on big data alone increases the chance that we'll miss something, while giving us this illusion that we already know everything.

Tricia Wang talks about the power of quantification models:

And what makes it really hard to see this paradox and even wrap our brains around it is that we have this thing that I call the quantification 
bias, which is the unconscious belief of valuing the measurable over the immeasurable.

And then it's on to addiction, to the self addicted to data:

But the problem is that quantifying is addictive. And when we forget that and when we don't have something to kind of keep that in check, it's very easy to just throw out data because it can't be expressed as a numerical value. It's very easy just to slip into silver-bullet thinking, as if some simple solution existed...There is no greater risk than being blind to the unknown. It can cause you to make the wrong decisions. It can cause you to miss something big.

And then its back to the oracle and the support the oracle gets from the 'temple guides':

...the temple guides would get to work...they would ask them follow-up questions, like, "Why do you want to know this prophecy? Who are you? What are you going to do with this information?" And then the temple guides would take this more ethnographic, this more qualitative information, and interpret the oracle's babblings. So the oracle didn't stand alone, and neither should our big data systems. 
Then Tricia Wang introduces the idea of 'thick data'. Is this a reference to Clifford Geertz's idea of 'thick understanding?

... in the same way that the oracle needed her temple guides, our big data systems need them, too. They need people like ethnographers and user researchers who can gather what I call thick data. This is precious data from humans, like stories, emotions and interactions that cannot be quantified... Thick data grounds our business questions in human questions, and that's why integrating big and thick data forms a more complete picture. Big data is able to offer insights at scale and leverage the best of machine intelligence, whereas thick data can help us rescue the context loss that comes from making big data usable, and leverage the best of human intelligence.

And then it's a cross reference to Netflix which we have considered in terms of Hastings and Meyer's book, No Rules Rules:

Now, when Netflix did this, they unlocked a whole new way to transform their business. Netflix is known for their really great recommendation algorithm, and they had this $\$ 1$ million prize for anyone who could improve it. And there were winners. But Netflix discovered the improvements were only incremental. So to really find out what was going on, they hired an ethnographer, Grant 
McCracken, to gather thick data insights. And what he discovered was something that they hadn't seen initially in the quantitative data. He discovered that people loved to binge-watch. In fact, people didn't even feel guilty about it. They enjoyed it.

So Netflix was like, "Oh. This is a new insight." So they went to their data science team, and they were able to scale this big data insight in with their quantitative data. And once they verified it and validated it, Netflix decided to do something very simple but impactful. They said, instead of offering the same show from different genres or more of the different shows from similar users, we'll just offer more of the same show. We'll make it easier for you to binge-watch. And they didn't stop there. They did all these things to redesign their entire viewer experience, to really encourage binge-watching. It's why people and friends disappear for whole weekends at a time, catching up on shows like "Master of None." By integrating big data and thick data, they not only improved their business, but they transformed how we consume media. And now their stocks are projected to double in the next few years.

And then Tricia Wang takes a wider sociological view:

But this isn't just about watching more videos or selling more smartphones. For some, integrating thick data insights into the algorithm could mean life or death, especially for the marginalized. All 
around the country, police departments are using big data for predictive policing, to set bond amounts and sentencing recommendations in ways that reinforce existing biases. NSA's Skynet machine learning algorithm has possibly aided in the deaths of thousands of civilians in Pakistan from misreading cellular device metadata. As all of our lives become more automated, from automobiles to health insurance or to employment, it is likely that all of us will be impacted by the quantification bias.

Next we might turn to Cathy O'Neill who suggests that the era of blind faith in big data must end. In an important, it might be suggested, a classic, Ted talk of 2017 Cathy O'Neill says:

Algorithms are everywhere. They sort and separate the winners from the losers...We're being scored with secret formulas that we don't understand that often don't have systems of appeal. That begs the question: What if the algorithms are wrong?

O'Neill describes the building of an algorithm as follows:

To build an algorithm you need two things: you need data, what happened in the past, and a definition of success, the thing you're looking for and often hoping for. You train an algorithm by looking, figuring out. The algorithm figures out 
what is associated with success. What situation leads to success?

Giving an example of algorithm use O'Neill talks about success and the value of opinion:

Actually, everyone uses algorithms. They just don't formalize them in written code. Let me give you an example. I use an algorithm every day to make a meal for my family. The data I use is the ingredients in my kitchen, the time I have, the ambition I have, and I curate that data.

My definition of success is: a meal is successful if my kids eat vegetables. It's very different from if my youngest son were in charge. He'd say success is if he gets to eat lots of Nutella. But I get to choose success. I am in charge. My opinion matters. That's the first rule of algorithms.

Algorithms are opinions embedded in code. It's really different from what you think most people think of algorithms. They think algorithms are objective and true and scientific. That's a marketing trick. It's also a marketing trick to intimidate you with algorithms, to make you trust and fear algorithms because you trust and fear mathematics. A lot can go wrong when we put blind faith in big data.

The idea that algorithms are opinions written in code could and perhaps should be considered in terms of the creation and maintenance of ideology, of political sentiments understood as truth. This 
goes with the obfuscation that this is all science and nothing but truth. O'Neill explains this through an example from the New York school system and then moves on to the example of Roger Ailes of Fox News and claims of sexual harassment to conclude:

\begin{abstract}
Algorithms don't make things fair if you just blithely, blindly apply algorithms...They repeat our past practices, our patterns. They automate the status quo... Because we all have bias, it means they could be codifying sexism or any other kind of bigotry.
\end{abstract}

Then, in another very telling expose of trust in algorithms O'Neill gives an example of how segregation is known. She goes on to conclude with a discussion of the phrases 'data laundering', 'weapons of math destruction' and 'secret sauce':

What's going on? Data laundering. It's a process by which technologists hide ugly truths inside black box algorithms and call them objective; call them meritocratic. When they're secret, important and destructive, I've coined a term for these algorithms: "weapons of math destruction."

They're everywhere, and it's not a mistake. These are private companies building private algorithms for private ends. Even the ones I talked about for teachers and the public police, those were built by private companies and sold to the government institutions. They call it their "secret sauce" -- that's 
why they can't tell us about it. It's also private power.

O’Neill offers another phrase, 'algorithmic audit', The good news is, we can check them for fairness. Algorithms can be interrogated, and they will tell us the truth every time. And we can fix them. We can make them better. I call this an algorithmic audit, and I'll walk you through it.

First, data integrity check. For the recidivism risk algorithm I talked about, a data integrity check would mean we'd have to come to terms with the fact that in the US, whites and blacks smoke pot at the same rate but blacks are far more likely to be arrested -- four or five times more likely, depending on the area. What is that bias looking like in other crime categories, and how do we account for it?

Second, we should think about the definition of success, audit that. Someone who stays for four years and is promoted once? Well, that is a successful employee, but it's also an employee that is supported by their culture. That said, also it can be quite biased. We need to separate those two things.

Next, we have to consider accuracy. No algorithm is perfect, of course, so we have to consider the errors of every algorithm. How often are there errors, and for whom does this model fail? What is the cost of that failure? 
And finally, we have to consider the long-term effects of algorithms, the feedback loops that are engendering. That sounds abstract, but imagine if Facebook engineers had considered that before they decided to show us only things that our friends had posted.

O'Neill concludes with a couple of messages and a warning about blind faith in big data:

I have two more messages, one for the data scientists out there. Data scientists: we should not be the arbiters of truth. We should be translators of ethical discussions that happen in larger society. And the rest of you, the non-data scientists: this is not a math test. This is a political fight. We need to demand accountability for our algorithmic overlords.

The era of blind faith in big data must end.

Cathy O'Neill is referenced in a very good paper on Cambridge Analytica, on May 24, 2017 in Ethnography Matters by Molly Sauter entitled Persuasion and the other thing: A critique of big data methodologies in politics. Molly Sauter's discussion of Cambridge Analytica in 2017 is considered in Six. Building on important work by Zeynep Tufcecki, Molly Sauter starts to talk about the modelling of individuals: 
In 2014, Tufekci described the information asymmetry problem of big data methodology, that it is not merely that subjects do not know as much about the researchers as the researchers know about them, but that as a core aspect of the methodology, subjects often do not even know they are being studied. While previous models of data collection allowed for the modeling of rough populations, Tufekci notes that big data analytics allow for the modeling of individuals without the researcher ever having to encounter that individual, or the individual being aware their actions are being taken into the political sphere.

In a continuation or extension of Cathy O'Neill's 'algorithmic audit' Molly Sauter argues:

It is reasonable for individuals to use tactics like obfuscation to resist attempts to be read, known, and manipulated as data objects.

Molly Sauter has a telling turn of phrase as she leads into a discussion of the machine readability of humans:

This is the "data doppelganger" (a term coined by critic Sara Marie Watson) overtaking the individual who is ostensibly its source, the echo overtaking the voice, the map overtaking the territory. In so much as these data doppels are used to directly impact, direct, and influence the lives of those individuals from whose actions they are derived, the sense in 
which their knowability is both assumed and constructed solely from building blocks provided by (powerful) others and rendered machine readable, they have a potential diminishing effect on these individuals' subjectivity and agency. The researcher (or the advertiser or campaign manager) is no longer dealing with a person possessed of their own self-determining agency and unmeasurable subjectivity, but rather manipulating a fully comprehensible data object.

Molly Sauter then turns to the model:

This model of grasping another person solely through pre-set categorizations and machinereadable actions means never being forced to encounter difference. Pre-established and machinereadable categories and actions are fundamentally aspects which are already familiar: they are recognized as important by the person collecting the data, hence, almost tautologically, their inclusion. But difference that breaches the bounds of the dataset becomes invisible.

Molly Sauter speaks of:

...The assumption of knowability, that a person can be grasped with mathematical completeness through their digital shadow-selves...

Then Sauter considers Facebook 'Likes' and their usages in putting together data pictures of people 
and after that to notions of persuasion and consent.

It is a short hop from thinking you know someone to thinking you know what they want or what is good for them, without any need to persuade or even to ask. And removing persuasion as a necessary step from the political sphere removes consent from the political sphere as well.

And in a way that speaks to the questions about governance throughout this Two Suns? series Sauter notes the risk in using these techniques in the political sphere,

This creates a national political fabric not of broad communities of multiple points of address and compromise to be governed holistically, but of schism-ed individuals and groups, each believing that they are the whole of the community that needs to be addressed, and anyone else is an interloper. Democracy shifts from a form of governance at least theoretically concerned with public debate and persuasion to one focused on private, opaque manipulation and emotional coercion.

The second risk occurs when politicians and governments, stuffed with psychographic data and algorithmic models, no longer feel the need to encounter the governed at all.

In an extremely important section of her work Molly Sauter asks: 
If we believe that widespread social-media-based big data modeling poses genuine risks to democracy, what is the best way to mitigate these risks?

Sauter goes on to give a wide ranging set of possible responses that an an individual might make in the face of Big Tech referring to work by Brunton and Nisselbaum inter alia:

Opting out, or social media abstinence immediately jumps to mind. However, this strategy is ineffective on a number of levels. First, it only protects those who are able to opt out from these tracking and modeling systems; given the central role social media plays in many people's social and professional lives, opting out is simply not a viable option for everyone. Second, regarding the specific issue of constituent modeling, opting out at an individual level would remove even the shadowrepresentation offered by the data doppelganger.

Obfuscation, as described by Finn Brunton and Helen Nissenbaum in their 2015 book of the same name, may be the best mode of resistance to pervasive surveillance and modeling systems that are unlikely to be rejected by those in power (or those who seek power) due to perceptions of their efficacy and profitability. By utilizing obfuscatory methods, Cambridge Analytica-style systems of constituent profiling and manipulation can be rendered ineffective for the targeted population as a whole, discouraging their use. 
...Several of these methods create noise, either at the level of the platform or the individual profile. Platform level noise generation might look like stacking a big data channel, like Twitter or Facebook, with noisy bots that share just enough characteristics with the targeted dataset to be included.

Another method, "like-farming," involves paying individuals to "like" products or brands on Facebook, often thousands at a time. This behavior could devalue "likes" as psychographic data.

At the individual obfuscation level, Brunton and Nissenbaum note several add-ons or experiments that operate on the logic of their TrackMeNot browser extension, which obfuscates an individual's genuine search history by generating a background hum of "fake" search requests for every "real" one.

AdNauseam works in the background of your web browser, invisibly clicking every ad on every page you visit. This activity floods ad tracking networks with useless and inaccurate data, and also allows those websites you visit to collect revenue from the pay-per-click ads they feature.

FaceCloak creates a network within the Facebook network, allowing users to store personal data with FaceCloak instead of Facebook. Users of the FaceCloak add-on can see your personal data as integrated with your Facebook page, but Facebook never possesses it.

Brunton and Nissenbaum also discuss "Bayesian flooding," which involves individuals actively 
feeding false information into their Facebook profiles: "The trick is to populate your Facebook with just enough lies as to destroy the value and compromise Facebook's ability to sell you" (Cho quoted in Brunton + Nissenbaum, p. 39).

One tactic that entails both individual and platform obfuscation is a patent held, interestingly enough, by Apple, entitled "Techniques to Pollute Electronic Profiling." Brunton and Nissenbaum describe it as a "cloning service," intended to "automate and augment the process of producing misleading personal information, targeting online data collectors." This cloning service would mimic a user's personal rhythms and behaviors, but "may begin to diverge from those interests in a gradual, incremental way," (p. 36) automatically browsing, clicking, signing up for websites and newsletters, chatting with other clones, maybe ordering small physical items from time to time. Eventually, in theory, attempting to find your "true" data stream among your mob of data clones would be like trying to find a needle in a haystack of other needles.

In what may be an important twist to the idea of the remote self offered by the present author (ibid) Molly Sauter speaks of 'mechanisms to distance the individual from the power of their vote':

As a modern democracy, the US excels in developing new mechanisms to distance the individual from the power of their vote. Big data methodologies and the inferential analytics they 
power as deployed in elections present yet another move to push people, in all their loud, messy, demanding changeability, out of politics. But unlike gerrymandering or the electoral college, this move can be actively resisted on the individual level.

Then there is the news and the situation of the person. If we turn to the Scott Morrison- Mark Zuckerberg standoff in Australia of early 2021, who is the individual to trust, Rupert Murdoch who is in the background encouraging and supporting Morrison from the point of view of 'old' media interests or Zuckerberg of Facebook? If we turn to Myanmar it gets more direct in that Facebook simply banned the government, a military government admittedly but the power of the land, so called. What does the individual in Myanmar know about their government if Facebook is the major supplier of news?

The examples from Myanmar and Australia show that for the individual it depends where you live. The variegation of rights across states especially between big and small nation states is striking and there for exploitation by the algorithmic state.

So much of the extant literature and commentary in this area like the Sackur-Lina Khan interview discussed in Six has a fairly tight focus on the USA, the UK and Europe. We might add a further set of points the first being China. Increasing numbers of sellers on Amazon, according to Dumaine's book on 
Bezonomics are Chinese. These third parties come in through another legal portal so to speak and are outside the US system. What does this mean for Lina Khan's support for such third party sellers? We might create a map with the points about infrastructure and space along with the identity of third party sellers in mind. We might now draw a map of Big Tech in various places around the globe, a job for an intrepid cartographer perhaps. The map might show $5 \mathrm{G}$ and facial recognition in China but not in the USA for example and variations of this type of thing around the world. Also in the map might be indications of acceptance or rejection of Big Tech itself.

To run over the map we might start by noting that Ireland gives tax breaks. Germany in 2019 insisted on a period before lock out of a seller. Vestager from the European Commission representing the countries in the EU offers a harder line to Big Tech. India, after a debate allowed Amazon to partner with a local firm. Australia is trying to make Google renegotiate with local advertisers. There is the lock out of certain social media platforms by China.

Also we might think about an altogether different form of mapping that might involve shells, hubs and populations. There is also a cartography of cultural difference to consider. I have been talking about the use of some of the anthropologist, Marshall Sahlin's ideas regarding high and low islands in Polynesia and the type of social organisation involved. 
There are general frames with an inclusive or exclusive distinction to consider. But this is shaded and varied. China includes $5 \mathrm{G}$ and facial recognition and excludes Google, Germany and the EU might exclude or monitor closely features on the Amazon market and yet include Alexa, the latter being critical in data collection.

Ireland might welcome it all.

Then there is the inversion of power descibed in Six and imagined in One and Two where a Big Tech company situates itself in a small country with the effect that the economic power iof the Big Tech operator massively exceeds that of the host state and that is discussed in the case of Baidu in the Cayman Islands in Six. Is this example portentous? Could we have a map where gigantic tech companies have tiny geographical footprints on the face of the earth? Would such a scenario be more advantageous to Big Tech companies than being corralled, so to speak in California?

How do these frames of reference, these cultural contours work?

Agency is another feature of any such cartography. So far nation-states are seen as key agents but then there is the lock out by Big Tech, the permanent suspension by Twitter of Donald Trump and others. Until, that is we get to Scomo and Mark, Morrison and Zuckerberg, Australia and Facebook. News reports on 22.02.21 were that Australians could not 
get vital news on things like climate because of the rift between the nation state of Australia and Facebook.

Then there is the lock out of tech by tech as with Amazon Web Services suspending Parler.

The episode of Black Mirror and self image considered in earlier parts of the Two Suns? series might be considered here. We are talking about identity, personal identity and citizenship in the algorithmic state. We could consider the reach of algorithmic state into personal space as well as other forms of space. This could take us to theorists of pleasure such as Huxley as well as theorists of television. We could talk about the dimensions of the algorithmic state and the dimensions of the self within it.

We are talking about a state in space, a state across spaces and the individual's role in this context, about their algorithmic fit. Or, after Sauter and O'Neil, about ways that individuals might fight the fit if they do not choose to wear it.

There is something of a strange transition here. It used to be the case and, to the unreflecting mind still is the case that eavesdropping and secret recordings were seen as abhorrent. But this has been replaced or supplanted by eavesdropping, as it were, in plain sight as with Alexa and other household listening and speaking devices. 
But its not to worry too much about the remote self, loneliness, fighting the algorithmic fit and the like as we have friends in the form of robots. We have cobotics, we have teamwork. In July, 2016 Janet Vertesi, in an article entitled 'What robots in space teach us about teamwork: A deep dive into NASA', talks about the machine- person interface. The sense of her article relates to increased teamwork in space but even in the five years since with developments like Scouts and many other robots or machines close to humans that this is happening elsewhere. As Vertesi notes early in her article in a section entitled 'One robot, many humans':

Robotic work is team work

To that end, working with a spacecraft is always teamwork, a creative task that brings together hundreds of people. Like any team, they use local norms of communication and interaction, and organizational routines and culture, in order to solve problems and achieve their goals. The spacecraft exploring our solar system have enough artificial intelligence to know better than to drive off a cliff, or they may know to reset their operating systems in case of a fault. There the autonomy ends. For the rest, every minute down to the second of their day is part of a plan, commanded and set into code by specialists on earth.

How to decide what the robot should do? First the team must take into account some basic constraints. When I studied the Mars Exploration 
Rover mission team, everyone knew that Opportunity could not drive very quickly; lately it has suffered from memory lapses and stiff joints in its old age. On another mission I have studied as an ethnographer, the path the spacecraft takes is decided years in advance to take into account the planetary system's delicate orbital dynamics and enable the team to see as much of the planet, its moons and rings as possible. It is not easy to change course. On all missions, limits of power, time, and memory on board matter provide hard constraints for planning.

Human factors are in the mix too. I often compare working on a spacecraft team to being on a bus with hundreds of people, each with their own idea about where to go and what to do - but with only one steering wheel. To make any decisions at all about robotic activities, the group first has to decide how to decide. They come up with a social organization for their team, codes of conduct and rules to govern their interactions. And they must constantly work together to prioritize which observations to send to the spacecraft.

When it comes to different kinds of teamwork one thinks of No Rules Rules the book by Hastings and Meyer discussed earlier and the look at cultures around the world in terms of the Netflix culture. What is the culture of cobotics found in this Big Tech firm or that, this country or that? Vertesi is talking about how things worjed in NASA half a decade ago and goes on to note: 
I have spent ten years researching robotic team decision making and I can say for certain that there is no one best way to command a spacecraft. One team I studied uses a matrix organization structure, sorting scientists associated with different instruments into cross-cutting working groups and charging those groups to decide what science should be done during different periods of the spacecraft's path. Another group I studied has a flat command structure and requires unilateral consensus across the whole team before the robot can act. I am analyzing these different groups in forthcoming work, comparing their work cultures and their organizational practices. Despite local differences, they have a lot in common. Each team is highly successful and conducts important scientific work. And even if they have different ways of working together, everyone on board is committed to reaching agreement.

But there is an effect to different strategies and and Vertesi argues:

Social organization affects the robot's actions ...As the decision-making process results in the robot conducting some scientific investigations but not others, this requires careful negotiation, difficult prioritization, and recourse to local cultures of decision-making to make sure everyone is on board with the plan. Ultimately, deciding how to decide plays an important role in how people relate to their robots. 
In an important section Vertesi asks how we organise with and around robots,

Organization and the robotic body

Organizations play another important role in the way that people relate to their robots. I saw this most clearly on the Mars Rover mission, where it was commonplace for team members to use their bodies to imitate the rover. They held their hands out to approximate the robot's camera "eyes," tilted their torsos back and forth to "feel" the pitch and roll of the craft, and swung their forearms awkwardly from their elbows like the robotic arm.

...this embodied activity helped rover team members to understand their robot's activities at a distance... Yet as everyone embodied the same robot together in the same way and felt its actions throughout their whole frame, this also reified their organization's commitment to unilateral consensus... The body work of being the rover also helped to turn the robot itself into the team's totem, which in turn cemented group solidarity and strong social ties.

Above Vertesi comes close to a 'method acting' approach Her work is very important as we imagine ourselves as robots and progress along a cobotic line to a point outlined in Five where we watch the robot perform and interact infrequently when things break down rather than, as Vertesi seems to be describing in her time of robot- human interaction, thinking of robots as extensions of ourselves. There 
is a mind shift going and Vertesi captures this even if her experience is a little dated,.

Robots, organizations, and design.

One way to move beyond the human-machine binary is to stop thinking only about one-humanone-machine. While it's tempting to imagine a future where we all have our own personal R2D2, humans are social beings. When robots join us at work, they will enter group settings with existing hierarchies, cultures, and interactional norms. From working on the shop floor, dispensing medication in a hospital, or cleaning our homes, these robots will need to navigate organizational norms alongside cultural expectations.

To that end, we must incorporate organizational thinking into our design thinking. This means investigating how different organizational forms demand different kinds of interactions from robotic agents. The intimacy of the home environment might influence how the Roomba is perceived like a pet or "one of the family," as Georgia Tech researchers found; meanwhile, a robot in a hospital might have a cheeriest bedside manner but it must know how to behave in a hierarchical workplace when nurses, doctors, and surgeons give contradictory orders.

We must also know enough about group work and organizational culture not to upset the delicate sociotechnical work that people do with their robots in our own design ambitions. Certainly a more perfect 3-D visualization of Mars might assist in 
robotic planning. But abandoning the shared body of the robot as a subject position may disrupt the important organizational work that the consensus team is doing on earth when they imagine their robots in space.

Participatory design may reveal ways forward as we enroll many stakeholders in design practice; ethnography can help to develop a vocabulary for expressing organizational routines and workarounds. But if there is one thing we can apply from NASA's robotic explorers to robotic workers of the future, it is to think organizationally about how they will join our human teams.

Vertesi's work seems to predate what is happening in Amazon's Fulfilment Centres where there seems to be little human involvement and in the Shanghai Example the reduction of human input to oversight rather than action shows this. The Vertesi article might speak of an early cobotic situation where robots are involved at the direction of humans while the Shanghai example discussed in Three and elsewhere in the Two Suns? series and that found in the Fulfilment Centres has humans in an inspectorate role rather than a participatory one.

\section{Conclusion}

The argument in the Two Suns? series might be summed up as follows. There are several spaces or forms of space involved; territorial space, outer space, cyberspace and living space. And there is 
data to be found and owned in each of these spaces. That data may be processed using algorithms in each space and across all spaces.

In this section, there has been the suggestion of a rephrasing of the self in the context of the algorithmic state. Not only is the count of Likes and the fear of being cut from a friends list the sort of thing that is now considered seriously there is also considerable variation of personal situations and rights from country to country.

We might now turn to data as a resource and consider the inequity of the situation perhaps best illustrated by Facebook, which takes but does not give data in an almost unimaginable scale. Again, we might have to go back to feudalism to look at a comparable gap between the public and the few.

People are caught up in the tech wars as with Parler and Amazon.

There are matters of agency. There are information angles, if you like, on the self where the person is fed data and, going back to Tricia Wang we might think of her example of the oracle. Or to Cathy O'Neill's thesis that data is structured and bent in ways that do not or at least do not necessarily relate to the interests of the self. Molly Sauter backs that up with her assessment of thick and other data and the effect of misreading data on marginalised people. Sauter also gives a very important and perhaps necessary set of coping mechanisms for the person fighting for identity in the Big Tech world. 
Our sense of team and teamwork is important here and Vertesi gives a perspective, which is a good one to begin an analysis of human, robot and cobot. Again, we come to volition and agency and back to the idea that there is a conservatism associated with the person in a data fed or cobotic situation. We could go back to the quote from DCB Pierre at the beginning of this section. Who would want to confound the software? 


\section{Bibliography}

Bleuca April 'Digital feudalism in a multipolar, unstable world' Responsible Statecraft 2021

Brunton, Finn Nissenbaum, Helen 2015 Obfuscation A User's Guide for Privacy and Protest MIT Press 2015

Dumaine, Brian Bezonomics How Amazon is changing our lives and what the world is learning from it, Simon and Schuster 2020

Geertz, Clifford, 'Thick Description: Toward an Interpretive Theory of Culture', in The Interpretation of Cultures: Selected Essays, Basic Books 1973 Hastings, Reed and Meyer, Erin No Rules Rules, Netflix and the Culture of Reinvention Virgin 2020 Huxley, Aldous Brave New World, Harper 1998 Huxley, Aldous Brave New World and Brave New World Revisited Harper 2004

Khan, Lina M. 'Amazon's Antitrust Paradox' The Yale Law Journal 2017 pp 710-805

O'Neill, Cathy Weapons of Math Destruction, Crown 2016

Pierre D B C Lights out in Wonderland, 2010 Faber and Faber

\section{Internet and Film Sources}

Cleave, Books and Books 31 Campus Press, Facebook

Sackur-Lina Khan interview 
Morrison and Zuckerberg, Australia and Facebook. News reports on 22.02.21

Amazon Sidewalk Terrifies Us - YouTube TWIT This Week in Tech Sep 30 2020https://www.youtube.com/watch?v=VasJplgi $\underline{3 z 8}$

Sauter, Molly May 24, 2017 Persuasion and the other thing: A critique of big data methodologies in politics in ETHNOGRAPHY MATTERS, Facebook The Rise of The Machines More than 100,000 Robots at Amazon Warehouse - YouTube Tricia Wang: The human insights missing from big data $\mid$ TED ...

https://www.ted.com , talks , transcript

19/07/2017

Zeynep Tufekci Big Questions for Social Media Big Data: Representativeness, Validity and Other Methodological Pitfalls [1403.7400] Big Questions for Social Media Big Data: Representativeness, Validity and Other Methodological Pitfalls (arxiv.org) Vertesi, Janet July, What robots in space teach us about teamwork: A deep dive into NASA | Ethnography Matters 